\title{
中国南方女性乳腺癌篮查“逸仙模式”初步 结果分析
}

\author{
杨雅平 ${ }^{\dagger}$, 梁静 $^{\dagger}$, 胡越, 申时雨, 顾然, 王红莉, 刘风桃, 梅静思, 蒋小芳, 云苗苗, \\ 龚畅
}

中山大学孙逸仙纪念医院乳腺肿瘤中心, 广东省恶性肿瘤表观遗传与基因调控重点实验室, 广州 510120

$\dagger$ 同等贡献

*联系人, E-mail: gchang@mail.sysu.edu.cn; changgong282@163.com

收稿日期：2020-06-19; 接受日期：2020-07-10; 网络版发表日期：2020-10-13

科技部国家重大研发计划(批准号：2017YFC1309103，2017YFC1309104)、国家自然科学基金面上项目(批准号：81672594，81772836, 81872139)、广州再生医学与健康广东省实验室临床创新研究项目(批准号：2018GZ0201004)、中山大学孙逸仙纪念医院逸仙临床研究培育 项目(批准号: SYS-C-201805)和逸仙科研起航项目(批准号: YXQH201920)资助

摘要乳房X线检查是目前唯一被证明可以降低乳腺癌死亡率的篎查方法. 然而, 乳房X线检查对年轻、乳房小 而致密的女性的检测准确度下降, 尤其在亚洲女性中较为常见. 因此, 本团队开展了中山大学孙逸仙纪念医院乳 腺篎查模式(简称“逸仙模式”), 通过联合运用乳腺超声和乳房X线, 对具有高乳腺密度和较高乳腺癌发病率的中 国南方地区女性进行乳腺癌䇻查. 本研究共纳入2013 2018年22 89岁参与南方职业女性乳腺癌䇻查项目的女性 28621 例。运用逸仙乳腺癌篎查模式, 即年龄 $\geq 40$ 岁参检者接受乳腺超声联合乳房 $\mathrm{X}$ 线检查, 年龄 $<40$ 岁参检者仅 接受乳腺超声检查, 并均进行为期 1 年的随访. 28621 名妇女共检出乳腺癌39例 $(1.36 / 1000)$. 其中，<40岁单一乳腺 超声检查组有6例乳腺癌(0.48/1000), ～４0岁乳腺超声联合乳腺X线检查组有33例 $(2.05 / 1000)$, 两者间差异具有统 计学意义 $(P<0.001)$. 在联合耖查模式组中, 仅有 8 例 $(24.2 \%)$ 仅通过乳房 $\mathrm{X}$ 线检测到癌症, 剩下 22 人 $(66.7 \%)$ 既可以 从超声检查中发现, 也可以从乳腺X线检查中发现. 大部分乳腺癌患者影像学表现仍是以肿块型为主, 但肿块型乳 腺癌和钙化型乳腺癌的比例在各个年龄段中无明显差异 $(P=0.632)$. 且对于钙化型乳腺癌, 目前仍是以乳腺 $\mathrm{X}$ 线发 现为主。就临床分期而言，0期及 I 期乳腺癌在单一乳腺超声组 $(66.7 \%, 4 / 6)$ 及联合篎查模式(66.7\%, 22/33)均超过 半数, 两组间比例差异无统计学意义 $(P=0.507)$. 中山大学逸仙乳腺癌筷查模式是一种可适用于中国南方地区女性 乳腺癌篎查的替代方法.

关键词乳腺癌, 篮查, 乳腺 $X$ 线, 乳腺超声

目前，乳腺癌已成为全球女性第一高发恶性肿瘤. 虽然欧美国家乳腺癌发病率居高, 但是随着以乳腺X
线摄影为基础的篎查模式的推广，及早发现、治疗, 其病死率逐年下降 ${ }^{[1,2]}$. 近些年来我国乳腺癌发病率明

引用格式: 杨雅平, 梁静, 胡越, 等. 中国南方女性乳腺癌笁查“逸仙模式”初步结果分析. 中国科学: 生命科学, 2020, 50: 1114-1120

Yang Y P, Liang J, Hu Y, et al. Preliminary results of the SYSU strategy for screening breast cancerin southern Chinese women (in Chinese). Sci Sin Vitae, 2020, 50: 1114-1120, doi: 10.1360/SSV-2020-0018 
显上升 ${ }^{[3]}$, 增速全球首位, 因此, 亟需建立并普及高效 的筷查模式. 与欧美女性相比, 大多数中国女性乳腺结 构具有较扁平而致密的特点, 而乳腺组织密度高的女 性患乳腺癌的风险更高, 癌症在乳房X线摄影检查中 被发现的可能性也更小 ${ }^{[4,5]}$. 我国的肿瘤登记资料显 示, 我国女性乳腺癌发病率在20岁后随年龄增长迅速 上升, 并于 55 岁年龄组达到高峰; 死亡率在 25 岁之后 上升迅速, 于 60 岁年龄组达到第 1 个高峰, 并于 85 岁以 上年龄组达到死亡高峰 ${ }^{[6]}$. 因此, 欧美国家针对自身发 病高峰年龄位于40 74岁的女性进行的基于乳腺X线 摄影的篎查模式 ${ }^{[2,7]}$ 并不完全适用于中国女性. 本研究 拟在2013 2018年28621例南方职业女性乳腺癌篮查 项目中, 根据年龄及检查方式制定中山大学乳腺癌篮 查模式, 并探讨其价值, 旨在构建一个适用于我国南 方女性的乳腺癌篮查模式.

\section{1 对象与方法}

\section{1 研究对象}

2013 2018年广州、珠海、佛山、中山等南方地 区事业单位参加团体性乳腺癌篎查的女性(含退休人 员), 共纳入 28621 例.

\section{2 研究方法}

针对年龄 $<40$ 岁的参检者, 仅予乳腺超声检查; 年 龄 $\geq 40$ 岁的参检者, 予乳腺 $X$ 线摄影及乳腺超声联合检 查, 均随访 1 年.

乳腺超声检查: 均由具有 3 年以上工作经验的超声 医生采用彩色多普勒超声仪进行全乳及区域淋巴结检 查, 并根据美国放射学会提出的2013版乳腺影像报告 和数据系统(Breast Imaging Reporting and Data System, BI-RADS $)^{[8]}$ 进行分级诊断 $(0$ 级: 需要进一步影像学检 查; 1 级: 阴性; 2 级: 良性; 3 级: 良性可能, 需短期随访; 4级: 可疑恶性, 建议活检; 5级: 恶性可能大; 6 级: 已病 理证实恶性).

乳腺X线摄影: 首先采用PLANMED平板数字乳 腺机, 以标准头足位(CC位)和内外斜位(MLO位)投照, 采集乳腺X线片, 再由两位经过相关培训的影像科医 生进行盲法阅片. 需描述乳腺腺体类型: 1型, 脂肪型; 2型, 少量腺体型; 3型, 多量腺体型; 4 型, 致密型 ${ }^{[9]}$, 并 根据BI-RADS进行分级诊断. 存在不统一的意见时, 需
讨论后达成一致.

\section{3 统计分析}

采用EpiData 3.1软件建立数据库, 双人平行双录 入并校对结果. 使用SPSS 23.0(IBM)软件进行数据整 理、统计描述和统计分析. 用描述性统计, 包括例数、 平均值、标准差、最大值和最小值来总结连续性变 量. 分类指标的描述用各类的例数及百分数. 连续性变 量采用两组独立样本的 $t$ 检验, 分类变量采用卡方检验 或Fisher精确概率法. 采用双侧检验, $P<0.05$ 认为有统 计学意义.

\section{2 结果}

\section{1 篮查人群的基本情况}

本研究共入组各企事业单位参加团体性篮选的女 性28621名, 平均年龄为 41.7 岁, 最小年龄为 22 岁, 最大 年龄为 89 岁. 本次研究篎查的人群中共检查发现乳腺 癌 39 人, 检出率为 $136.3 / 10$ 万. 检查乳腺癌人群的平均 年龄为 47.5 岁, 最小年龄为 31 岁, 最大年龄为 64 岁.

由表1可见, 从 30 岁组开始就有女性被检出乳腺 癌, 检出乳腺癌的最小年龄是31岁. 随着年龄的增加, 特别是35岁以后, 乳腺癌篮查人群恶性情况的检出率 逐渐增加, 55岁组的检出率最高, 达到 $375.4 / 10$ 万人; 其次是 50 岁组的女性, 检出率为 $241.6 / 10$ 万人; 从 60 岁 组开始有所降低, 但是也有 $205.5 / 10$ 万人. 本次筛查中, 65 岁以上年龄组的女性并未发现乳腺癌, 结果详见 图1.

\section{2 不同年龄组篮查出乳腺癌人群基本情况的 比较}

本研究中, $<40$ 岁的女性仅做乳腺超声检查, 共篮 查 12515 名女性, 检查乳腺癌 6 名, 检出率为 $47.9 / 10$ 万 人. 在 $\geq 40$ 岁组人群乳腺癌篎查时既做了乳腺超声检 查, 也做了乳腺X线检查, 共筛查16106名女性, 检查乳 腺癌 33 名, 检出率为 $204.9 / 10$ 万人. 两个年龄组的检出 率有差别, $\geq 40$ 岁组人群的检出率更高, $P<0.001$. 虽然 $<40$ 岁年龄组女性总的检出率较低, 但是在35 岁组仅 做乳腺超声组和40 岁组同时做乳腺超声和乳腺X线 检查的两组中, 检出率差别并不大 $(109.1 / 10$ 万 $v s$. $169.1 / 10$ 万). < 40 岁组女性确诊乳腺癌的平均年龄为 
表 1 乳腺篎查人群不同年龄组检出恶性的情况

Table 1 The malignant conditions identified among the different age groups via the SYSU strategy

\begin{tabular}{|c|c|c|c|c|c|}
\hline 年龄组(岁) & 笁查人数 & 各年龄组比例 & 笁查确诊恶性人数 & 年龄组恶性比例 & 各年龄组检出率(/10万) \\
\hline 平均年龄 & $41.7 \pm 10.6(22 \sim 89)$ & & $47.5 \pm 7.1(31 \sim 64)$ & & \\
\hline$<30$ & 3612 & 12.6 & 0 & 0.0 & 0.0 \\
\hline $30 \sim$ & 4322 & 15.1 & 1 & 2.6 & 23.1 \\
\hline $35 \sim$ & 4581 & 16.0 & 5 & 12.8 & 109.1 \\
\hline $40 \sim$ & 5322 & 18.6 & 9 & 23.1 & 169.1 \\
\hline $45 \sim$ & 4744 & 16.6 & 10 & 25.6 & 210.8 \\
\hline $50 \sim$ & 2897 & 10.1 & 7 & 18.0 & 241.6 \\
\hline $55 \sim$ & 1332 & 4.7 & 5 & 12.8 & 375.4 \\
\hline $60 \sim$ & 973 & 3.4 & 2 & 5.1 & 205.5 \\
\hline $65 \sim$ & 491 & 1.7 & 0 & 0 & 0.0 \\
\hline $70 \sim$ & 347 & 1.2 & 0 & 0 & 0.0 \\
\hline 合计 & 28621 & 100 & 39 & 100 & 136.3 \\
\hline
\end{tabular}

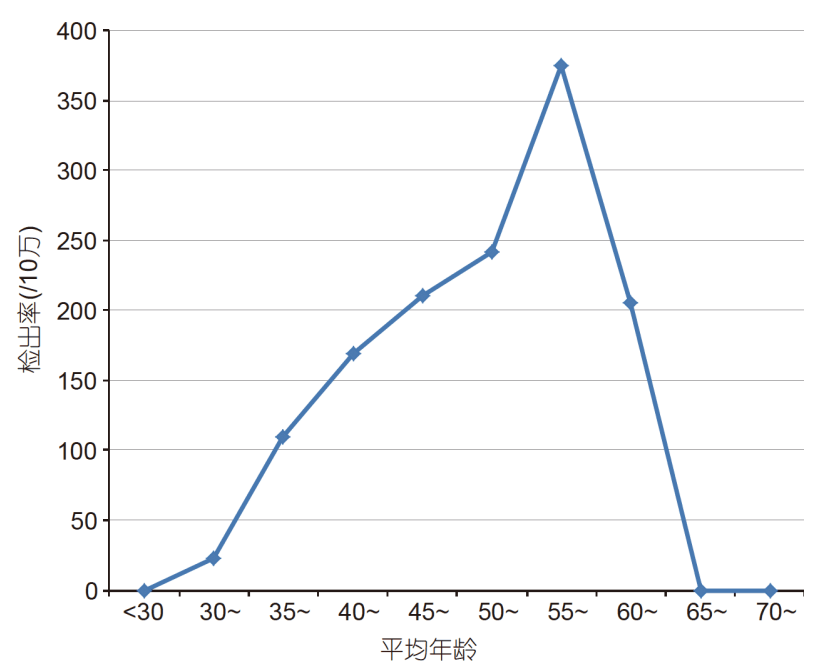

图 1 乳腺癌篎查人群各年龄组恶性情况检出率(网络版 彩图)

Figure 1 The detection rate of malignancy in each age group of breast cancer screening among the women population (color online)

(37 \pm 3.2$)$ 岁(31 39岁); 而 $\geq 40$ 岁组女性确诊乳腺癌的平 均年龄为(49 \pm .9) 岁(41 64岁), 两组差异为12岁, 且差 异有统计学意义, $P<0.001$.

从发现方式来看, 因为 $<40$ 岁组女性仅做了乳腺 超声，因此乳腺癌的检出均是超声发现的. 然而, $\geq 40$ 岁组女性中, 有 3 人 $(9.1 \%)$ 是仅从超声检查发现的乳 腺癌恶性征象，有 8 人 $(24.2 \%)$ 是仅从乳腺X线发现的 恶性征象，剩下 22 人 $(66.7 \%)$ 既可以从超声检查中发
现, 也可以从乳腺X线检查中发现. 结果详见图2和 表2.

\section{3 不同年龄组篮查出乳腺癌人群临床病理分期 特征的比较}

两个年龄组确诊乳腺癌的女性中, 病理类型的分 布情况是相似的, 均是以浸润性导管癌为主, 其次是 导管原位癌. $<40$ 岁组确诊恶性的女性中, 浸润性导管 癌的比例为 $66.7 \%(4 / 6)$, 导管原位癌的比例为 $33.3 \%$ $(2 / 6)$. 在 $\geq 40$ 岁组女性中, 浸润性导管癌的比例也是 $66.7 \%(22 / 33)$, 导管原位癌的比例为 $24.2 \%(8 / 33)$, 还有 3 例是其他浸润性癌(包括 1 例浸润性小叶癌和 2 例黏液 癌), 两组结果差异不显著, $P=0.755$, 详见表 2 .

临床分期方面，笁查发现的39例乳腺癌患者中，0 期(导管原位癌)为 10 例, I 期为 16 例, II 期为 8 例, III期 为 5 例, 其中 $0 \sim \mathrm{I}$ 期的比例占 $66.7 \%$. 两个年龄组临床 分期情况的分布可见, 0 期和 I 期的比例均是最高的 $(0$ 期 $33.3 \%$ vs. 24.2\%，I 期 $33.3 \%$ vs. 42.4\%); III期的比例 在 $<40$ 岁为 $33.3 \%$, 而在 $\geq 40$ 岁组仅为 $9.1 \%$, 但是两组各 分期的比例差别不大, $P=0.258$, 无统计学意义.

\section{4 不同年龄组耖查出乳腺癌人群的影像学特征 比较}

$<40$ 岁组女性中, 确诊恶性的患者都是以乳腺肿块 的形式, 而40 岁组、50 岁组和60 岁组乳腺恶性肿 


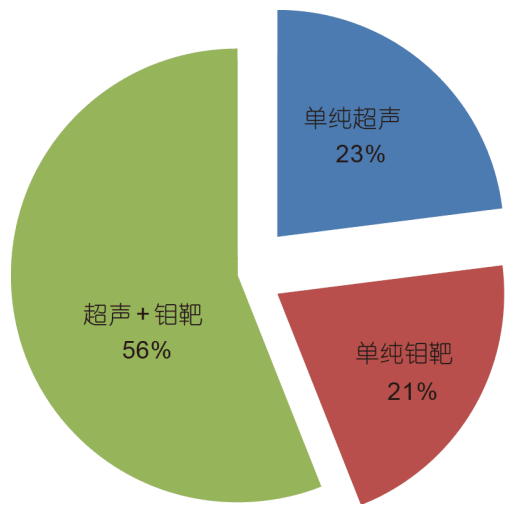

图 2 乳腺癌䇻查不同发现方式的分布图(网络版彩图)

Figure 2 Distribution of the different ways of identifying breast cancer screening (color online)

瘤的表现形式肿块和钙化均有，其中肿块型病变的比 例较高, 均在 $70 \%$ 以上, 钻化形式的病变比例大约在 $20 \%$ 25\%左右．但是在各年龄组之间，肿块型病变和 钙化型病变不同比例的差异，没有统计学意义 $(P=0.632)$. 不同年龄组不同篮查项目发现比例有一定 差别, <40岁组女性均是以乳腺超声的检查手段篮查 发现的. 以钻化形式存在的病灶, 还是以乳腺X线检查 发现为主，而部分肿块型病灶既可以用乳腺X线发现 也可以用超声检查发现, $P<0.001$, 差异有统计学意义 (表3).

\section{3 讨论}

目前，乳腺X线检查是唯一一种被证明可以降低 乳腺癌死亡率的篮查方式 ${ }^{[10]}$. 研究表明, 虽然乳腺X线 可以显著降低50 74岁女性乳腺癌死亡率，但在 40 49 岁女性中的作用却十分有限, 这可能与其随着乳腺密 度的增加, 敏感性显著下降有关 ${ }^{[1]}$, 且其对极高密度乳 房的敏感性可能低至 $30 \% \sim 48 \%{ }^{[4]}$. 因此, 诸多研究试图 通过联合篮查模式提高乳腺癌的检出率 ${ }^{[11 ~ 13]}$. 超声因 其广泛可用、价格相对便宜、患者的耐受性好备受关 注, 成为一种很有前景的篮查方式.

本研究通过中山大学乳腺癌笁查模式, 总乳腺癌 检出率可达 $136.3 / 10$ 万人, 是国际年龄标准化乳腺癌 发病率 $40.8 / 10$ 万人 ${ }^{[14]}$ 的3倍，与西方发达国家发病率 相接近, 也明显高于国内平均水平 ${ }^{[6,15]}$, 说明本乳腺癌 笁查模式检出率水平较高. 本实验结果显示, 乳腺癌人 群的平均发病年龄为 47.5 岁, 与既往研究结果基本持 平, 早于西方发达国家近 10 年 ${ }^{[3,16]}$. 通过进一步比较不 同年龄组的乳腺检出率，可以发现30岁后即开始有女 性被诊断出乳腺癌, 随后发病率逐渐增高, 高峰期接 近西方发达国家，大约50 55岁，这一结果也与既往我 国女性乳腺癌发病特点分析相符 ${ }^{[6]}$. 日本有研究曾对 比乳腺彩超联合乳腺 $X$ 线篮查模式和单一乳腺 $X$ 线检

表 2 不同年龄组笁查出乳腺癌人群基本情况的比较

Table 2 Comparison of the basic conditions of the women with breast cancer in different age groups

\begin{tabular}{|c|c|c|c|c|c|c|}
\hline \multirow{2}{*}{ 变量 } & \multirow{2}{*}{ 分组 } & \multicolumn{2}{|c|}{$<40$ 岁 } & \multicolumn{2}{|c|}{$\geq 40$ 岁 } & \multirow{2}{*}{$P$ 值 } \\
\hline & & 例数 $(N)$ & 百分比(\%) & 例数 $(N)$ & 百分比(\%) & \\
\hline 合计 & & 6 & & 33 & & \\
\hline 检出率 & & $6 / 12515$ & 47.9/10万 & $33 / 16106$ & 204.9/10万 & $<0.001$ \\
\hline 年龄 & & $37 \pm 3.2(31 \sim 39)$ & & $49 \pm 5.9(41 \sim 64)$ & & $<0.001$ \\
\hline \multirow{3}{*}{ 发现方式 } & 单纯超声 & 6 & 100.0 & 3 & 9.1 & $<0.001$ \\
\hline & 单纯钼靶 & 0 & 0 & 8 & 24.2 & \\
\hline & 超声+钼靶 & 0 & 0 & 22 & 66.7 & \\
\hline \multirow{3}{*}{ 病理类型 } & 导管原位癌 & 2 & 33.3 & 8 & 24.2 & 0.755 \\
\hline & 浸润性导管癌 & 4 & 66.7 & 22 & 66.7 & \\
\hline & 其他浸润性癌 & 0 & 0 & 3 & 9.1 & \\
\hline \multirow{4}{*}{ 临床分期 } & 0 期 & 2 & 33.3 & 8 & 24.2 & 0.258 \\
\hline & I 期 & 2 & 33.3 & 14 & 42.4 & \\
\hline & II 期 & 0 & 0 & 8 & 24.2 & \\
\hline & III期 & 2 & 33.3 & 3 & 9.1 & \\
\hline
\end{tabular}


表 3 不同年龄组笁查出乳腺癌人群的影像学特征

Table 3 Comparison of the imaging features of the women with breast cancer in different age groups

\begin{tabular}{|c|c|c|c|c|c|c|c|c|}
\hline 年龄组(岁) & 笁查人数 & 乳腺癌患者 & $\begin{array}{l}\text { 肿块 } \\
N(\%)\end{array}$ & $\begin{array}{l}\text { 钲化 } \\
N(\%)\end{array}$ & $\begin{array}{c}\text { 超声发现 } \\
N(\%)\end{array}$ & $\begin{array}{c}\text { 钼靶发现 } \\
N(\%)\end{array}$ & $\begin{array}{c}\text { 超声+钼靶 } \\
N(\%)\end{array}$ & $\begin{array}{c}\text { 各年龄组检出率 } \\
(/ 10 \text { 万 })\end{array}$ \\
\hline$<40$ & 12515 & 6 & $6(100.0)$ & $0(0.0)$ & $6(100.0)$ & $0(0.0)$ & $0(0.0)$ & 47.9 \\
\hline $40 \sim$ & 10066 & 19 & $14(73.7)$ & $5(26.3)$ & $3(15.8)$ & $5(26.3)$ & 11(57.9) & 188.8 \\
\hline $50 \sim$ & 4229 & 12 & $9(75.0)$ & $3(25.0)$ & $0(0.0)$ & $3(25.0)$ & $9(75.0)$ & 283.8 \\
\hline $60 \sim$ & 1811 & 2 & $2(100.0)$ & $0(0.0)$ & $0(0.0)$ & $0(0.0)$ & $2(100.0)$ & 110.4 \\
\hline 合计 & 28621 & 39 & $31(79.5)$ & $8(20.5)$ & $9(23.1)$ & $8(20.5)$ & $22(56.4)$ & 136.3 \\
\hline$P$ 值 & & & 0.632 & & & $<0.001$ & & \\
\hline
\end{tabular}

查篎查模式，发现联合篎查模式检出率明显高于单一 乳腺X线篮查模式 ${ }^{[12]}$. 本研究中, 乳腺彩超联合乳腺X 线篮查模式检出率可达 $204.9 / 10$ 万人, 高于国内类似回 顾性研究 ${ }^{[15]}$, 且不同笁查模式的敏感度分析结果显示, 乳腺彩超联合乳腺X线检查敏感度比单一乳腺彩超及 单一乳腺X线检查均明显升高 ${ }^{[1]}$, 这也与本研究的结 果相符. 因此, 有理由相信, 乳腺彩超联合乳腺X线检 查可作为新的乳腺癌笁查模式加以推广. 更有意思的 是, 虽然 $\geq 40$ 岁组人群乳腺彩超联合乳腺 $X$ 线检查的检 出率明显高于 $<40$ 岁单一乳腺彩超，但是在35 岁仅做 乳腺超声组和 40 岁乳腺超声联合乳腺X线检查组的 检出率却没有明显差异，可见 $<40$ 岁单一乳腺彩超检 出率低可能是受年龄因素影响, 且已有研究显示, 单 一乳腺彩超与单一乳腺X线检查敏感度基本一致 ${ }^{[11]}$. 此外, 本研究通过分析乳腺癌患者的影像学表现发现, 40 岁组、50 岁组和60 岁年龄组的乳腺癌患者大部 分是以肿块型为主，而 $<40$ 岁的乳腺癌患者则均是肿
块型，因此，乳腺超声检查作为对乳腺肿块病灶较为 敏感的检查方式在女性乳腺筱查中发挥重要作用, 尤 其在 $<40$ 岁女性中实行单一乳腺超声检查具有较好的 检出价值. 本研究对于钙化型乳腺癌病灶的发现, 仍主 要依赖乳腺X线检查，且部分肿块型乳腺癌既可以通 过乳腺彩超发现, 也能通过乳腺X线发现, 因此乳腺X 线检查仍是女性乳腺癌篮查中必不可少的检查方式, 且乳腺 $\mathrm{X}$ 线联合乳腺超声后，检出率更是显著提高 $(P<0.001)$. 就乳腺癌病理分期特点, 本团队发现, 不论 哪个发病年龄段, 均是以导管癌为主, 且临床分期均以 0 I 期占比最多, 这也与国外及国内其他回顾性研究 结果相似 ${ }^{[16,17]}$.

综上，本研究在 $<40$ 岁组女性中运用乳腺超声篮 查, 在 $\geq 40$ 岁组女性中运用乳腺超声联合乳腺X线检查 的篮查——乳腺癌逸仙篮查模式, 均能够有效进行篮 查. 逸仙乳腺癌笁查模式可成为适合中国南方地区女 性乳腺癌耖查的替代方法.

\section{参考文献}

1 Lauby-Secretan B, Scoccianti C, Loomis D, et al. Iarc working group briefing on breast cancer screening. Chin Gener Pract, 2015, 18: 3265-3267

2 Nickson C, Velentzis L, Brennan P, et al. Improving breast cancer screening in Australia: a public health perspective. Public Health Res Pract, 2019,29

3 Wu C, Li M, Meng H, et al. Analysis of status and countermeasures of cancer incidence and mortality in China. Sci China Life Sci, 2019, 62: $640-647$

4 Mandelson M T, Oestreicher N, Porter P L, et al. Breast density as a predictor of mammographic detection: comparison of interval- and screendetected cancers. J Natl Cancer Inst, 2000, 92: 1081-1087

5 Day N, Warren R. Mammographic screening and mammographic patterns. Breast Cancer Res, 2000, 2: 247-251

6 Li H, Zheng R S, Zhang S W, et al. Analysis on the incidence and death of breast cancer in Chinese women in 2014 (in Chinese). Chin J Oncol, 2018, 40: 166-171 [李贺, 郑荣寿, 张思维, 等. 2014年中国女性乳腺癌发病与死亡分析. 中华肿瘤杂志, 2018, 40: 166-171]

7 Wernli K J, Arao R F, Hubbard R A, et al. Change in breast cancer screening intervals since the 2009 USPSTF guideline. J Womens Health, 2017, 
26: $820-827$

8 Mendelson E B, Böhm-Vélez M, Berg W A, et al. ACR BI-RADS ultrasound. In: ACR BIRADS Atlas, Breast Imaging Reporting and Data System. 5th Ed. Reston: American College of Radiology. 2013

9 Kerlikowske K, Miglioretti D L, Vachon C M. Discussions of dense breasts, breast cancer risk, and screening choices in 2019. JAMA, 2019, 322: 69

10 Nelson H D, Tyne K, Naik A, et al. Screening for breast cancer: Systematic evidence review update for the U.S. preventive services task force. Ann Intern Med, 2009, 10: 242-727

11 Huzarski T, Górecka-Szyld B, Huzarska J, et al. Screening with magnetic resonance imaging, mammography and ultrasound in women at average and intermediate risk of breast cancer. Hered Cancer Clin Pract, 2017, 15: 4

12 Ohuchi N, Suzuki A, Sobue T, et al. Sensitivity and specificity of mammography and adjunctive ultrasonography to screen for breast cancer in the Japan Strategic Anti-cancer Randomized Trial (J-START): a randomised controlled trial. Lancet, 2016, 387: 341-348

13 Dang Y, Guo L, Lv D J, et al. Classification of breast lesions based on a dual S-shaped logistic model in dynamic contrast enhanced magnetic resonance imaging. Sci China Life Sci, 2011, 54: 889-896

14 Ferlay J, Soerjomataram I, Dikshit R, et al. Cancer incidence and mortality worldwide: Sources, methods and major patterns in GLOBOCAN 2012. Int J Cancer, 2015, 136: E359-E386

15 Zeng F Y, Zhang S, Tang G R, et al. Analysis of breast cancer screening results in 11167 women in Guilin (in Chinese). Chin J General Surg, 2012, 21: 509-510 [曾繁余, 张珊, 唐桂荣, 等. 桂林市11167名妇女乳腺癌篮查结果分析. 中国普通外科杂志, 2012, 21: 509-510]

16 Li J, Zhang B N, Fan J H, et al. A nation-wide multicenter 10-year (1999-2008) retrospective clinical epidemiological study of female breast cancer in China. BMC Cancer, 2011, 11: 364

17 Cho N, Han W, Han B K, et al. Breast cancer screening with mammography plus ultrasonography or magnetic resonance imaging in women 50 years or younger at diagnosis and treated with breast conservation therapy. JAMA Oncol, 2017, 3: 1495-1502 


\title{
Preliminary results of the SYSU strategy for screening breast cancerin southern Chinese women
}

\author{
YANG YaPing, LIANG Jing, HU Yue, SHEN ShiYu, GU Ran, WANG HongLi, LIU FengTao, \\ MEI JingSi, JIANG XiaoFang, YUN MiaoMiao \& GONG Chang \\ Guangdong Provincial Key Laboratory of Malignant Tumor Epigenetics and Gene Regulation, Breast Tumor Center, Sun Yat-sen Memorial Hospital, \\ Sun Yat-sen University, Guangzhou 510120, China
}

Mammography is the only screening method that has been found to reduce the mortality of breast cancer. However, it has been reported to be inaccurate in young women or women with small and dense breasts, a feature that is very common in Asian women. This study investigated a novel breast cancer screening strategy (SYSU strategy) based on ultrasonography and mammography for Southern Chinese women who have a high proportion of dense breasts and a high prevalence in young breast cancer. A total of 28,621 women aged 22-89 years were enrolled in this study between 2013-2018. They all attended the Southern Professional Women Breast Cancer Screening Project with an organized breast cancer screening. The SYSU breast cancer screening strategy involves participants who are older than 40 years being screened by ultrasound and mammography and those younger than 40 years screened by ultrasound alone at enrolment and 1-year follow-up. A total of 39 breast cancers were detected during the screening period (1.36/1000). Among the cancers detected, 6 breast cancers $(0.48 / 1000)$ were identified by ultrasound alone in women younger than 40 years, and 33 cancers $(2.05 / 1000)$ were identified by the combination of ultrasound and mammography $(P<0.001)$. However, in the combined group, only 8 cancers $(24.2 \%)$ were detected by mammography alone, while 22 other types of cancers $(66.7 \%)$ were detected by both mammography and ultrasound images. More than half of the cancers at stage 0 and I were detected in both the ultrasound group $(66.7 \%, 4 / 6)$ and combined group $(66.7 \%, 22 / 33)$, respectively, and the difference of proportion between the two groups was not significant $(P=0.507)$. The results of this study suggest that the SYSU strategy is a suitable and alternative method for screening breast cancers in Southern Chinese women.

breast cancer, screening, mammography, ultrasonography

doi: $10.1360 / \mathrm{SSV}-2020-0018$ 